\title{
EFFECT OF AMC SIDEWALLS STRUCTURES IN PARALLEL PLATE SLOT ANTENNAS
}

\author{
J.M. Fernández , M. Sierra Castañer
}

\begin{abstract}
Electromagnetic BandGap (EBG) structures have unique properties in controlling the propagation of electromagnetic waves. In this work we analyse the effect of EBG sidewalls acting as artificial magnetic conductors (AMC) sidewalls, first in a parallel plate waveguide and as practical application in parallel plate slot antennas. These structures present some interesting properties that may overcome some of the problems of conventional technologies. The results of the effect using AMC in comparison with perfect electric conductor (PEC) in the sidewalls of the parallel plate slot antennas is presented as examples of application. Using AMC sidewalls in this kind of antennas, relatively uniform field distributions are improved, so it allows to increase the directivity to enhance the efficiency of these antennas.
\end{abstract}

\section{INTRODUCTION}

The Electromagnetic bandgap (EBG) structures are novel periodic structures that, by generating a bandgap, can properly control the propagation of electromagnetic waves. These structures can be one, two or three dimensional periodic structures and they have been investigated for their versatility in controlling the propagation of electromagnetic waves in one, two or three dimensions. EBG technology presents some interesting properties that may overcome some of the problems of conventional technologies. In particular, recently, several EBG structures have been proposed and demonstrated to be useful in enhancing performances of microwave circuits or antennas. Therefore, in this paper, we are going to show that the problem of the non-uniform field distribution in a parallel plate waveguide with PEC sidewalls can be prevent using artificial magnetic conductor (AMC) sidewalls. To date, AMC surfaces are receiving more and more attention since they offer advantages that cannot be accomplished by utilizing traditional PEC. In contrast to the realization of a PEC, which is not difficult in practical situations, the realization of an AMC remains a difficult task. The difficulty stems from the fact that no suitable material has been found which can be used as a PMC. One possibility to design an AMC is to use EBG periodic structures [1].

Previous published papers have analysed standard rectangular waveguide with EBG structures acting as PMC sidewalls to finds application in designing novel TEM waveguide [2], without considering the degradation of the field causing non-uniform field distribution. This work differs from previously published contributions because EBG structures acting as AMC have been applied in the 
sidewalls of a parallel plate waveguide and parallel plate slot waveguides in order to provide an uniform field distribution inside the waveguide. A most uniformity of the field distributions inside this kind of antennas allows us to increase the directivity, getting to enhance the efficiency of these antennas. These practical applications will allow us to validate the simulation results

The EBG structure that has been used as AMC sidewalls is based on [1]. This uniplanar periodic 2D EBG structure is particularly attractive and has been intensively investigated due to their advantage of being simple, compact size, low cost and can be easily fabricated using any standard planar process without using any extra multilayer substrates or vias holes, which are necessary for other types of EBG structures. This 2D EBG structure will be applied to solve the problem showed in [3]

\section{ANALYSIS OF PARALLEL PLATE WAVEGUIDE}

The prototype of the waveguide that we analysed is a rectangular parallel plate waveguide of $330 \mathrm{~mm} \times 318 \mathrm{~mm}$ with sidewalls, between that we had a foam dielectric of $7.5 \mathrm{~mm}$ height as shown in Fig. 1. The operating frequency of this waveguide is $11.92 \mathrm{GHz}$. The ideal functioning of the waveguide only propagates a TEM mode (or quasi TEM). A rectangular waveguide-fed feed slots excite the parallel plate waveguide to generate a TEM mode as shown in Fig. 2. A common characteristic to the propagation of this kind of mode is its degradation at the same time as we move away from the feed point that causes non-uniform field distributions in the waveguide with PEC sidewalls.

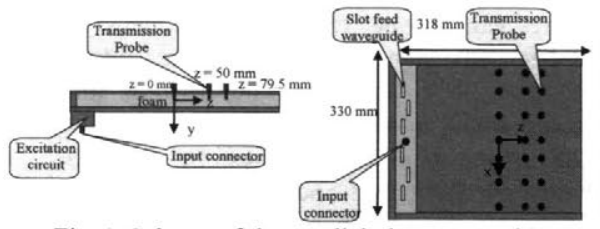

Fig. 1. Scheme of the parallel plate waveguide

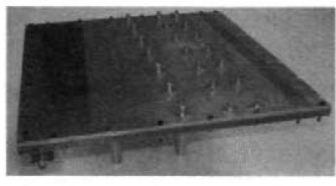

Fig. 2. Experimental prototype

In Fig. 3 and Fig. 4, the disposition of the 2D EBG structure in the prototype of parallel plate waveguide is shown.

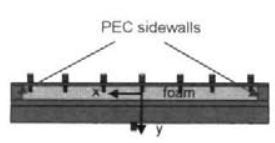

Fig. 3. Profile parallel plate waveguide with PEC sidewalls

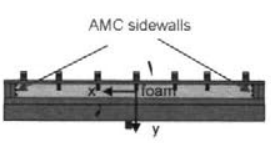

Fig. 4. Profile parallel plate waveguide with AMC sidewalls

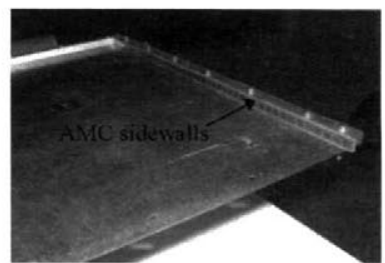

Fig. 5. Inner parallel plate waveguide with EBG structure acting as AMC sidewalls 
Fig. 5 shows the disposition of the prototyped 2D uniplanar EBG structure that behaves like an AMC structure. Each element of this EBG lattice consists of a square metal pad with four connecting branches.

The field profile distributions in the parallel plate waveguide with PEC and AMC sidewalls have been simulated and measured at the frequency of $11.92 \mathrm{GHz}$.

Fig. 6 shows the comparison of the simulated and the experimental results of the transmission coefficients in the parallel plate waveguide with PEC sidewalls and in Fig. 7 with AMC sidewalls. Good agreement between the simulations and the measurements are observed.

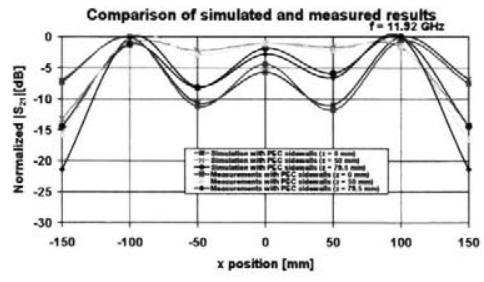

Fig. 6. Comparison results of the transmission coefficients in the parallel plate waveguide with PEC sidewalls

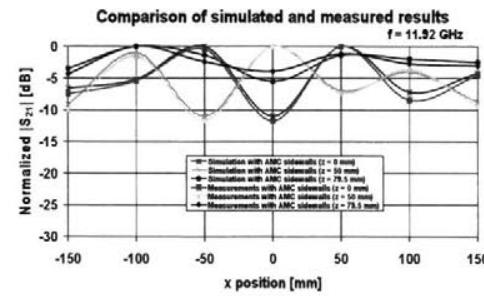

Fig. 7. Comparison results of the transmission coefficients in the parallel plate waveguide with AMC sidewalls

The ripples that can be seen in the results are due to that the TEM wave has been generated with the array of slot in the feed waveguide. These ripples tend to be smooth at the same time as we move away from the rectangular waveguide-fed feed slots in the parallel plate waveguide.

As can be seen in Fig. 7, the AMC sidewalls waveguide has a relatively uniform field distribution compared to the standard waveguide with PEC sidewalls.

One thing that should be pointed out here is that the peak value of the field in the PMC sidewalls waveguide is lower than that in the PEC sidewalls waveguide due to the field flattening.

\section{PRACTICAL APPLICATIONS}

The 2D uniplanar EBG structure acting as AMC is applied in parallel plate slot antennas with linear polarization shown in Fig. 8 [4], which operating frequency is $12 \mathrm{GHz}$.

This kind of antennas is a rectangular parallel plate waveguide excited by rectangular waveguide-fed slots as shown in Fig. 8 [3]. This excitation generates a TEM plane wave that propagates in the perpendicular direction of the axis of the parallel plate waveguide. This plane wave excites the radiating structure that consists of a planar array of resonant slots in the upper plate of the waveguide. 


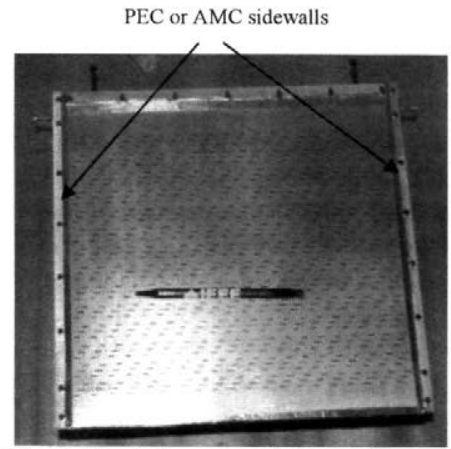

Fig. 8. Single beam parallel plate slot antenna with linear polarization excited by rectangular waveguide.

\begin{tabular}{|c|c|c|}
\hline $\mathrm{f}=12 \mathrm{GHz}$ & \multicolumn{2}{|c|}{ Directivity } \\
\hline & PEC & AMC \\
\hline $\begin{array}{c}\text { Single beam } \\
\text { parallel } \\
\text { plate slot } \\
\text { antenna }\end{array}$ & $27.3 \mathrm{dBi}$ & $27.6 \mathrm{dBi}$ \\
\hline
\end{tabular}

Fig. 9. Experimental results using AMC sidewalls in the single beam parallel plate slot antenna

\section{CONCLUSIONS}

The simulation and experimental analysis effects using EBG sidewalls acting as AMC in a parallel plate waveguide and in parallel plate slot antennas have been presented. The results show that a fairly uniform field distribution along the transverse direction of the parallel plate waveguide with AMC sidewalls has been obtained. These results demonstrate the feasibility of applying PMC sidewalls to improve the characteristics of parallel plate slot antennas. A most uniformity of the field distributions inside the waveguide allow us to increase the directivity, to enhance the efficiency of these kinds of antennas. These practical applications will allows us to validate the simulation results. In Fig. 9, experimental results are shown. We observed the directivity improvement effect of the AMC sidewalls in the single beam parallel plate slot antenna shown in Fig. 8.

\section{REFERENCES:}

[1] K.-P. Ma ,K. Hirose, F.-R. Yang, Y. Quian and T. Itoh, "Realization of magnetic conducting surface using novel photonic bandgap structure", IEE Electronic Letters, Vol. 34, $\mathrm{N}^{\circ}$ 21, pp. 2041-2042, October 1998.

[2] F.-R. Yang, K.-P. Ma, Y. Qian and T. Itoh, "A novel TEM waveguide using uniplanar compact photonic-bandgap (UC-PBG) structure", IEEE Trans. Microwave Theory and Techniques, Vol. 47, N 11, pp.2092-2098, November 1999.

[3] H. Kai, J. Hirokawa and M. Ando, "Field distribution in multi-mode rectangular waveguides" IEEE Transactions on Antennas and Propagation, Vol. 1, pp.110-113, July 2000

[4] M. Sierra Castañer, J. Izquierdo, M. Sierra Pérez, J.L. Fernández, M. Vera "Linear polarization parallel plate spot antenna", IEEE AP-S Symposium, Vol. 3, pp.16081611, Salt Lake City, July 2000. 